\title{
Video Grading: Giving Students Personal Feedback in an Online World
}

\author{
Andrew J Donadio, ${ }^{*}$ Sheila Hurley \\ Tennessee Tech University, USA
}

\section{Editorial}

Engaging students in a distance learning environment can be difficult. One method that can help professors bridge the gap is making the use of video grading. Grading via video note is an alternative to traditional grading of careplans, discussion boards and other assignments. The goal is two-fold: to enhance the student experience, bringing the online world to a less impersonal level andprovide feedback with less ambiguity helping students attain a better grasp of course content quickly. Utilization of this method does require some individual technical knowledge of your learning management system (LMS). This technique is successful on both CANVAS and Desire2 Learn. A Zoom account is also helpful (Zoom Pro not required). It is also recommended you have a quality webcam and microphone so the audio and video quality is acceptable.

Using Zoom (or similar program), start a meeting and share the student assignment on your screen- this is easier using two monitors. If using Zoom, the professor will appear in the upper corner as guidance. This adds a personal touch to the feedback bridging the distance gap common to the online environment since you are speaking directly to each student.

To ensure success, the professor should have a comfort level with the camera and institution LMS. The feedback received from students is almost always positive and during COVID this engagement has been important for students who are normally on-ground. Video grading can also add personalization to any online course.

\begin{tabular}{|c|l|}
\hline \hline Quick Response Code: & FCorresponding author: Andrew J Donadio, Tennessee Tech University, 910 Franklin Ct, USA \\
Received: 28 May, 2021 Published: 04 Jun, 2021 \\
Citation: Andrew JD, Sheila H. Video Grading: Giving Students Personal Feedback in an Online \\
World. Trends Nur Health Care Res. 2021;1(2):1-1. DOI: 10.53902/TNHCR.2021.01.000506 \\
\hline
\end{tabular}

